\title{
Modified Legendre Collocation Block Method for Solving Initial Value Problems of First Order Ordinary Differential Equations
}

\author{
Toyin Gideon Okedayo, Ayodele Olakiitan Owolanke, Olaseni Taiwo Amumeji, \\ Muyiwa Philip Adesuyi \\ Department of Mathematics, Ondo State University of Science and Technology, Okitipupa, Nigeria \\ Email: okedayo@yahoo.com
}

How to cite this paper: Okedayo, T.G., Owolanke, A.O., Amumeji, O.T. and Adesuyi, M.P. (2018) Modified Legendre Collocation Block Method for Solving Initial Value Problems of First Order Ordinary Differential Equations. Open Access Library Journal, 5: e4565.

https://doi.org/10.4236/oalib.1104565

Received: June 15, 2018

Accepted: July 3, 2018

Published: July 6, 2018

Copyright $\odot 2018$ by authors and Open Access Library Inc.

This work is licensed under the Creative

Commons Attribution International

License (CC BY 4.0).

http://creativecommons.org/licenses/by/4.0/

(c) (1) Open Access

\begin{abstract}
In this paper, block procedure for some k-step linear multi-step methods, using the Legendre polynomials as the basis functions, is proposed. Discrete methods were given which were used in block and implemented for solving the initial value problems, being continuous interpolant derived and collocated at grid points. Some numerical examples of ordinary differential equations were solved using the derived methods to show their validity and the accuracy. The numerical results obtained show that the proposed method can also be efficient in solving such problems.
\end{abstract}

\section{Subject Areas \\ Mathematical Analysis}

\section{Keywords}

Block, Legendre Polynomials, Zero-Stable, Convergent

\section{Introduction}

Many problems in celestial and quantum mechanics, nuclear, theoretical physics, astrophysics, quantum chemistry and molecular dynamics, are of great interest to scientists and engineers. These problems are mathematically modelled by using ordinary differential equation of the form:

$$
f\left(x, y, y^{\prime}, y^{\prime \prime}, \cdots, y^{(n)}\right), y(a)=y_{0}, y^{\prime}(a)=y_{1}, \cdots, y^{(n-1)}(a)=y_{n}
$$

where on the interval $[a, b]$ has given rise to two major discrete variable methods namely, one step and multistep methods commonly known as linear mul- 
ti-step methods. Many authors have worked on the direct solution of (1), among which are Lambert [1], Fatunla [2], Sarafyan [3], Awoyemi [4] and Kayode [5]. Each of them worked on the development of several methods for solving Equation (1) directly without having to reduce to system of first order differential equations. For instance, in Awoyemi [4], methods were developed to solve second order initial value problems which are the mathematical formulation for systems without dissipation. Fatunla [2] considered a step-by-step method based on the classical Runge-Kutta method; Hairer and Wanner [6] developed Nystrom type method for initial value problem for first order differential equations in which the conditions for the determination of the parameters of the methods were mentioned. Also, Henrici [7] and Lambert [1] improved the derivation of linear multi-step methods with constant coefficients for solving first order equation with initial conditions.

In Awoyemi [8], linear multi-step methods with continuous coefficient for initial value problem of the first order differential equations in the predictor-corrector mode were proposed, based on collocation method with power series polynomial as basis function, and Taylor series algorithm to supply starting values. Continuous linear multi-step method is useful in reducing the step number of a method and still remains zero-stable; it has greater advantage in the sense that better error estimates guaranteed easy approximation of solution to all points of integration interval. Moreover, Awoyemi (1995) adopted the hybrid methods and proposed a two-step hybrid multi-step method with continuous coefficients for the solution of a first order initial value problem based on the collocation at selected grid points, using off-grid points to improve the order of the method implemented on the predictor-corrector mode. Other researchers who have studied hybrid method include Adee and Onumanyi [9], and Yahaya and Badmus [10].

Furthermore, many researchers had developed interest on improving the numerical solution of initial value problems of ordinary differential equation. Consequently, the development of a class of methods called block method is one of the outcomes. This was proposed by Milne [11], and it was found that it generates approximations continuously at different grid points in the interval of integration; it is less expensive in terms of the number of function evaluations compared to the linear multi-step methods. Chu and Hamilton [12] also proposed a generalization of the linear multi-step method to a class of multi-block methods where step values are obtained all together in a single block. Jator (2007) and Jator et al. (2005) proposed five-step and four-step self starting methods which adopt continuous linear multi-step method to obtain finite difference method applied respectively as a block for the direct solution of the first order initial value problem. Also, in Yahaya and Mohammed (2010), Chebyshev polynomial was considered as trial function. Ajileye et al. adopted Laguerre collocation approach for continuous hybrid block method. Other scholars that adopted block methods include Omar and Suleiman [13] [14] [15] and Areo and 
Adeniyi [16]. Abualnaja (2015) developed a block procedure with linear multi-steps using Legendre polynomials but did not include the block schemes. Thus, in this paper, Legendre polynomial is used as a basis function to derive some block methods for the solution of first order ordinary differential equation, which extends the work of Abualnaja (2005).

\section{Derivation of the Method}

In this section, we consider the approximate solution of the form

$$
y_{k}(x)=\sum_{i=0}^{k} c_{i} \psi_{i}(x) .
$$

Perturbing the equation above, we have

$$
\sum_{i=0}^{k} c_{i} \psi_{i}(x)=f(x, y)+\lambda L_{k}(x)
$$

where, $\psi_{i}(x)=x^{i}, i=0,1, \cdots, k$ and $L_{k}(x)$ is the Legendre polynomial of degree $k$, which is defined on the interval $[-1,1]$, and can be determined with the aid of the recurrence formula:

$$
L_{i+1}(x)=\frac{2 i+1}{i+1} x L_{i}(x)-\frac{i}{i+1} L_{i-1}(x), i=1,2, \cdots
$$

So that

$$
L_{0}(x)=1, L_{1}(x)=x, L_{2}(x)=\frac{3 x^{2}-1}{2}, L_{3}(x)=\frac{5 x^{3}-3 x}{2}, L_{4}(x)=\frac{35 x^{4}-30 x^{2}+3}{2}
$$

We define a shifted Legendre polynomials by introducing the change of variable

$$
x=\frac{2 \bar{x}-\left(x_{n+k}+x_{n}\right)}{x_{n+k}-x_{n}}, k=1,2,3,4
$$

For $k=1$

using Equation (4), taking $L_{1}(x)=x$, and collocating at $x_{n}$ and $x_{n+1}$, we obtain

$$
L_{1}\left(x_{n}\right)=-1, L_{1}\left(x_{n+1}\right)=1
$$

hence,

$$
L_{1}\left(x_{n}\right)=\frac{2 x_{n}-x_{n+1}-x_{n}}{x_{n+1}-x_{n}}=-1
$$

and,

$$
L_{1}\left(x_{n+1}\right)=\frac{2 x_{n+1}-x_{n+1}-x_{n}}{x_{n+1}-x_{n}}=1
$$

Deducing $\psi_{0}(x)=0, \psi_{1}(x)=1$ from Equation (1), it follows that Equation (2) becomes

$$
f(x, y)=c_{1}-\lambda L_{1}(x)
$$

Solving the above systems we obtain

$$
\lambda=\frac{1}{2}\left(f_{n}-f_{n+1}\right), c_{1}=f_{n}-\lambda, c_{0}=y_{n}-x_{n}\left(f_{n}-\lambda\right)
$$


The required numerical scheme of the method will be obtained if we collocate $y(x)=c_{0}+c_{1} x$ at $x=x_{n+1}$ and substitute $c_{0}, c_{1}, \lambda$ as follows

$$
y_{n+1}=y_{n}+\frac{h}{2}\left(f_{n+2}+f_{n}\right)
$$

$k=2$

taking $L_{2}(x)=\frac{1}{2}\left(3 x^{2}-1\right)$, and collocating at $x_{n}, x_{n+1}$, and $x_{n+2}$

we get

$$
L_{2}\left(x_{n}\right)=1, L_{2}\left(x_{n+1}\right)=\frac{-1}{2}, L_{2}\left(x_{n+2}\right)=1
$$

From Equation (1), it can be deduced that $\psi_{0}(x)=0, \psi_{1}(x)=1, \psi_{2}(x)=2 x$, then Equation (2) becomes

$$
f(x, y)=c_{1}+2 x c_{2}-\lambda L_{2}(x)
$$

Collocating Equation (10) at $x_{n+i}, i=0,1,2$ and interpolate

$$
y_{k}(x)=\sum_{i=0}^{k} c_{i} \psi_{i}(x), x_{n} \leq x \leq x_{n+k}
$$

at $x=x_{n}$, we get a system of four equations with $c_{i}(i=0,1,2)$ and parameter $\lambda$

$$
\begin{aligned}
& y_{n}=c_{0}+c_{1} x_{n}+c_{2} x_{n}^{2} \\
& f_{n}=c_{1}+2 c_{2} x_{n}-\lambda \\
& f_{n+1}=c_{1}+2 c_{2} x_{n+1}-\frac{1}{2} \lambda \\
& f_{n}=c_{1}+2 c_{2} x_{n+2}-\lambda
\end{aligned}
$$

Hence, solving Equation (12), we get

$$
\begin{aligned}
& \lambda=\frac{1}{3}\left(-f_{n}+2 f_{n+1}-f_{n+2}\right) \\
& c_{0}=\frac{-1}{12 h}\left(-12 h y_{n}+8 t_{n} h f_{n+1}-4 t_{n} h f_{n+2}+8 t_{n} h f_{n}-3 t_{n}^{2} f_{n+2}+3 t_{n}^{2} f_{n}\right) \\
& c_{1}=\frac{1}{6 h}\left(4 h f_{n+1}-2 h f_{n+2}+4 h f_{n}-3 t_{n} f_{n+2}+3 t_{n} f_{n}\right) \\
& c_{2}=\frac{-1}{4 h}\left(f_{n}-f_{n+2}\right)
\end{aligned}
$$

From $y_{k}(x)=\sum_{i=0}^{k} c_{i} \psi_{i}(x)$, we have

$$
\bar{y}=c_{0}+c_{1} x+c_{2} x^{2}
$$

Hence, the required numerical scheme is obtained by collocating Equation (14) above at $x=x_{n+1}$ and substituting $c_{0}, c_{1}, c_{2}, \lambda$ as follows

$$
y_{n+1}=y_{n}+\frac{h}{12}\left(5 f_{n}+8 f_{n+1}-f_{n+2}\right)
$$

$k=3$

Taking the polynomial $L_{3}=\frac{1}{2}\left(5 x^{3}-3 x\right)$ and use the Equation (4), then collocating this at $x_{n}, x_{n+1}, x_{n+2}$ and $x_{n+3}$, we obtain 
$L_{3}\left(x_{n}\right)=-1, \quad L_{3}\left(x_{n+1}\right)=\frac{11}{27}, L_{3}\left(x_{n+2}\right)=\frac{-11}{27}, L_{3}\left(x_{n+3}\right)=1$. From Equation

(1), it can be deduced that $\psi_{0}(x)=0, \psi_{1}(x)=1, \psi_{2}(x)=2 x, \psi_{3}(x)=3 x^{2}$, then Equation (2) is reduced to the form

$$
f(x, y)=c_{1}+2 x c_{2}+3 c_{3} x^{2}-\lambda L_{3}(x)
$$

Hence, collocating Equation (16) at $x_{n+i}, i=0,1,2,3$ and interpolate Equation (11) at $x=x_{n}$, we get the system of equations with $c_{i},(i=0,1,2,3)$ and parameter $\lambda$

$$
\begin{aligned}
& y_{n}=c_{0}+c_{1} x_{n}+c_{2} x_{n}^{2}+c_{3} x_{n}^{3} \\
& f_{n}=c_{1}+2 c_{2} x_{n}+3 c_{3} x_{n}^{2}+\lambda \\
& f_{n+1}=c_{1}+2 c_{2} x_{n+2}+3 c_{3} x_{n+1}^{2}-\frac{11}{27} \lambda \\
& f_{n+2}=c_{1}+2 c_{2} x_{n+2}+3 c_{3} x_{n+2}^{2}+\frac{11}{27} \lambda \\
& f_{n+3}=c_{1}+2 c_{2} x_{n+3}+3 c_{3} x_{n+3}^{2}-\lambda
\end{aligned}
$$

Solving the above system of equations, we obtain

$$
\begin{gathered}
\lambda=\frac{9}{40}\left(f_{n}-3 f_{n+1}+3 f_{n+2}-f_{n+3}\right) \\
c_{0}=-\frac{1}{120}\left(-81 t_{n} h^{2} f_{n+2}+27 t_{n} f_{n+3} h^{2}+56 t_{n}^{2} h^{2} f_{n}+81 t_{n} h^{2} f_{n+1}+93 t_{n} h^{2} f_{n}-18 t_{n}^{2} h f_{n+1}\right. \\
\left.+34 t_{n}^{2} h f_{n+3}-72 t_{n}^{2} h f_{n+2}-120 t_{n}^{2} h f_{n+2}-10 t_{n}^{3} f_{n+2}+10 t_{n}^{3} h f_{n+3}-10 t_{n}^{3} f_{n+1}\right) \\
c_{1}=\frac{1}{120}\left(68 t_{n} h f_{n+3}+112 t_{n} f_{n} h-36 t_{n}^{2} h f_{n+1}-144 t_{n} h f_{n+2}+81 h^{2} f_{n+1}+27 h^{2} f_{n+3}\right. \\
\left.+93 h^{2} h f_{n}-81 h^{2} f_{n+2}-30 t_{n}^{2} f_{n+2}+30 t_{n}^{2} f_{n+3}+30 t_{n}^{2} f_{n}-30 t_{n}^{2} f_{n+1}\right) \\
c_{2}=-\frac{1}{60 h^{2}}\left(-15 t_{n} f_{n+2}+15 t_{n} f_{n}-15 t_{n} f_{n+1}-36 h f_{n+2}+28 h f_{n}+17 h f_{n+3}-9 h f_{n+1}\right) \\
c_{3}=-\frac{1}{12 h^{2}}\left(f_{n}-f_{n+1}-f_{n+2}+f_{n+3}\right)
\end{gathered}
$$

From $y_{k}(x)=\sum_{i=0}^{k} c_{i} \psi_{i}(x)$, we have

$$
\bar{y}=c_{0}+c_{1} x+c_{2} x^{2}+c_{3} x^{3}
$$

Hence, the required numerical scheme is obtained by collocating Equation (18) above at $x=x_{n+1}$ and substituting $c_{0}, c_{1}, c_{2}, c_{3}, \lambda$ as follows

$$
y_{n+1}=y_{n}+\frac{h}{120}\left(47 f_{n}+89 f_{n+1}-19 f_{n+2}+3 f_{n+3}\right)
$$

$k=4$

In this case, we take the polynomial $L_{4}=\frac{1}{8}\left(35 x^{4}-30 x^{2}+3\right)$ and use the Equation (4), then collocating this at $x_{n}, x_{n+1}, x_{n+2}, x_{n+3}$ and $x_{n+4}$, we obtain $L_{4}\left(x_{n}\right)=1, L_{4}\left(x_{n+1}\right)=\frac{-37}{128}, L_{4}\left(x_{n+3}\right)=\frac{3}{8}, L_{4}\left(x_{n+4}\right)=1$. From Equation (1), 
we can deduce that $\psi_{0}(x)=0, \psi_{1}(x)=1, \psi_{2}(x)=2 x, \psi_{3}(x)=3 x^{2}, \psi_{4}(x)=4 x^{3}$, then Equation (2) is reduced to the form

$$
f(x, y)=c_{1}+2 x c_{2}+3 c_{3} x^{2}+4 c_{4} x^{3}-\lambda L_{4}(x)
$$

Hence, collocating Equation (20) at $x_{n+i}, i=0,1,2,3,4$ and interpolate Equation (11) at $x=x_{n}$, we get the system of equations with $c_{i},(i=0,1,2,3,4)$ and parameter $\lambda$

$$
\begin{aligned}
& y_{n}=c_{0}+c_{1} x_{n}+c_{2} x_{n}^{2}+c_{3} x_{n}^{3}+c_{4} x_{n}^{4} \\
& f_{n}=c_{1}+2 c_{2} x_{n}+3 c_{3} x_{n}^{2}+4 c_{4} x_{n}^{3}-\lambda \\
& f_{n+1}=c_{1}+2 c_{2} x_{n+1}+3 c_{3} x_{n+1}^{2}+4 c_{4} x_{n+1}^{3}+\frac{37}{128} \lambda \\
& f_{n+2}=c_{1}+2 c_{2} x_{n+2}+3 c_{3} x_{n+2}^{2}+4 c_{4} x_{n+2}^{3}-\frac{3}{8} \lambda \\
& f_{n+3}=c_{1}+2 c_{2} x_{n+3}+3 c_{3} x_{n+3}^{2}+4 c_{4} x_{n+3}^{3}+\frac{37}{128} \lambda \\
& f_{n+4}=c_{1}+2 c_{2} x_{n+4}+3 c_{3} x_{n+4}^{2}+4 c_{4} x_{n+3}^{3}-\lambda
\end{aligned}
$$

Solving the above system of equations with a suitable method, we obtain

$$
\begin{gathered}
\lambda=\frac{16}{105}\left(-f_{n}+4 f_{n+1}-6 f_{n+2}+4 f_{n+3}-f_{n+4}\right) \\
c_{0}=-\frac{1}{5040 h^{3}}\left(768 x_{n} h^{3} f_{n+4}-4272 x_{n} f_{n} h^{3}-3072 x_{n} h^{3} f_{n+3}\right. \\
\quad-3072 x_{n} h^{3} f_{n+1}+4608 x_{n} h^{3} f_{n+2}+2400 x_{n}^{2} h^{2} f_{n+1}-3330 x_{n}^{2} h^{2} f_{n} \\
+1290 x_{n}^{2} h^{2} f_{n+4}-4320 x_{n}^{2} h^{2} f_{n+3}+1520 x_{n}^{3} h f_{n+1}-1840 x_{n}^{3} h f_{n+3} \\
\quad+670 x_{n}^{3} h f_{n+4}-1010 x_{n}^{3} h f_{n}+660 x_{n}^{3} h f_{n+2}+3960 x_{n}^{2} h^{2} f_{n+2} \\
\left.+5040 y_{n} h^{3}+210 x_{n}^{4} f_{n+1}-210 x_{n}^{4} f_{n+3}+105 x_{n}^{4} f_{n+4}-105 x_{n}^{4} f_{n}\right) \\
c_{1}=\frac{1}{840 h^{3}}\left(-1320 x_{n} h^{2} f_{n+2}-330 x_{n}^{2} f_{n+2} h+505 x_{n}^{2} f_{n} h-335 x_{n}^{2} f_{n+4} h\right. \\
+920 x_{n}^{2} f_{n+3} h+760 x_{n}^{2} h f_{n+1}+1440 x_{n} h^{2} f_{n+3}-430 x_{n} h^{2} f_{n+4}+1110 x_{n} h^{2} f_{n} \\
+800 h^{2} x_{n} f_{n+1}-128 h^{3} h f_{n+4}-768 h^{3} h f_{n+2}+512 h^{3} f_{n+1}+512 h^{3} f_{n+3} \\
\left.+712 h^{3} f_{n}-140 x_{n}^{3} f_{n+1}+140 x_{n}^{3} f_{n+3}-70 x_{n}^{3} f_{n+4}+70 x_{n}^{3} f_{n}\right) \\
c_{2}=-\frac{1}{168 h^{3}}\left(-66 x_{n} h f_{n+2}+101 x_{n} h f_{n}-67 x_{n} h f_{n+4}+184 x_{n} h f_{n+3}-152 x_{n} h f_{n+1}\right. \\
+43 h^{2} f_{n+4}+111 f_{n} h^{2}-42 x_{n}^{2} f_{n+1}+42 x_{n}^{2} f_{n+1}+42 x_{n}^{2} f_{n+3} \\
\left.-21 x_{n}^{2} f_{n+4}+21 x_{n}^{2} f_{n}-80 h^{2} f_{n+1}+144 h^{2} f_{n+3}-132 h^{2} f_{n+3}\right) \\
c_{3}=-\frac{1}{504 h^{3}}\left(-84 x_{n} f_{n+1}+84 x_{n} f_{n+3}-42 x_{n} f_{n+4}+42 x_{n} f_{n}\right. \\
\left.\quad-152 h f_{n+1}+184 h f_{n+3}-67 h f_{n+4}+101 h f_{n}-66 h f_{n+2}\right) \\
c_{4}=-\frac{1}{48 h^{3}}\left(f_{n}-2 f_{n+1}+2 f_{n+3}-f_{n+4}\right)
\end{gathered}
$$

From $y_{k}(x)=\sum_{i=0}^{k} c_{i} \psi_{i}(x)$, we have 


$$
\bar{y}=c_{0}+c_{1} x+c_{2} x^{2}+c_{3} x^{3}+c_{4} x^{4}
$$

Hence, the required numerical scheme is obtained by collocating Equation (22) above at $x=x_{n+1}$ and substituting $c_{0}, c_{1}, c_{2}, c_{3}, c_{4}, \lambda$ as follows

$$
y_{n+1}=y_{n}+\frac{h}{5040}\left(1847 f_{n}+4162 f_{n+1}-1308 f_{n+2}+382 f_{n+3}-43 f_{n+4}\right)
$$

\section{Formulating the Block Scheme of Cases $k=2,3$ and 4}

If $k=2$

We collocate Equation (14) at $x=x_{n+1}, x_{n+2}, x_{n+3}$ to give us

$$
\begin{aligned}
& y_{n+1}=y_{n}+\frac{h}{12}\left(5 f_{n}+8 f_{n+1}-f_{n+2}\right) \\
& y_{n+2}=y_{n}+\frac{h}{3}\left(f_{n}+4 f_{n+1}+f_{n+2}\right) \\
& y_{n+3}=y_{n}+\frac{h}{4}\left(8 f_{n+1}-f_{n}+f_{n+2}\right)
\end{aligned}
$$

If $k=3$

We collocate Equation (18) at $x=x_{n+1}, x_{n+2}, x_{n+3}, x_{n+4}$ to give us

$$
\begin{aligned}
& y_{n+1}=y_{n}+\frac{h}{120}\left(47 f_{n}+89 f_{n+1}-19 f_{n+2}+3 f_{n+3}\right) \\
& y_{n+2}=y_{n}+\frac{h}{60}\left(21 f_{n}+77 f_{n+1}+23 f_{n+2}-f_{n+3}\right) \\
& y_{n+3}=y_{n}+\frac{h}{8}\left(3 f_{n}+9 f_{n+1}+9 f_{n+2}+3 f_{n+3}\right) \\
& y_{n+4}=y_{n}+\frac{h}{30}\left(29 f_{n}-7 f_{n+1}+47 f_{n+2}+51 f_{n+3}\right)
\end{aligned}
$$

If $k=4$

We collocate Equation (22) at $x=x_{n+1}, x_{n+2}, x_{n+3}, x_{n+4}, x_{n+5}$ to give us

$$
\begin{aligned}
& y_{n+1}=y_{n}+\frac{h}{5040}\left(1847 f_{n}+4162 f_{n+1}-1308 f_{n+2}+382 f_{n+3}-43 f_{n+4}\right) \\
& y_{n+2}=y_{n}+\frac{h}{90}\left(29 f_{n}+124 f_{n+1}+24 f_{n+2}+4 f_{n+3}-f_{n+4}\right) \\
& y_{n+3}=y_{n}+\frac{h}{560}\left(179 f_{n}+377 f_{n+1}+444 f_{n+2}+334 f_{n+3}-31 f_{n+4}\right) \\
& y_{n+4}=y_{n}+\frac{h}{45}\left(14 f_{n}+64 f_{n+1}+24 f_{n+2}+64 f_{n+3}+14 f_{n+4}\right) \\
& y_{n+5}=y_{n}+\frac{h}{1008}\left(-253 f_{n}+3322 f_{n+1}-1308 f_{n+2}+1222 f_{n+3}+2057 f_{n+4}\right)
\end{aligned}
$$

\section{Basic Properties of the Method}

\subsection{Order, Error Constant and Consistency of the Methods}

The schemes developed belong to the class of Linear Multi-step Method (LMM) of the form

$$
\sum_{j=0}^{k} \alpha_{j}(x) y\left(x_{n+j}\right)=h \sum_{j=0}^{k} \beta_{j}(x) f\left(x_{n+j}\right)
$$

Equation (27) is a method associated with a linear difference operator 


$$
L[y(x) ; h]=\sum_{j=0}^{k}\left(\alpha_{j} y(x+j h)=h \beta_{j} y^{\prime}(x+j h)\right)
$$

where $y(x)$ is continuously differentiable on the interval $[a, b]$, and the Taylor series expansion about the point $x$ is expressed as

$$
L[y(x) ; h]=c_{0} y(x)+c_{1} h y^{\prime}(x)+c_{2} h^{2} y^{\prime \prime}(x)+\cdots+c_{q} h^{q} y^{q}(x)
$$

In line with [12], schemes $(15,19,23)$ are said to be of order $P$ if $C_{0}=C_{1}=C_{2}=\cdots=C_{p}=0$ and the error constant is $C_{p+1} \neq 0$. Hence, we establish that (15),(19), and (23) is of the following orders respectively

when $k=2, P=3$ and $C_{p+1}=0.041667$

when $k=3, P=3$ and $C_{p+1}=0.016$

when $k=4, P=2$ and $C_{p+1}=0.041667$

\subsection{Stability Analysis}

The scheme can be expressed as:

$$
\begin{aligned}
{\left[\begin{array}{lll}
1 & 0 & 0 \\
0 & 1 & 0 \\
0 & 0 & 1
\end{array}\right]\left[\begin{array}{l}
y_{n+1} \\
y_{n+2} \\
y_{n+3}
\end{array}\right]=} & {\left[\begin{array}{lll}
0 & 0 & 1 \\
0 & 0 & 1 \\
0 & 0 & 1
\end{array}\right]\left[\begin{array}{c}
y_{n-2} \\
y_{n-1} \\
y_{n}
\end{array}\right]+h\left[\begin{array}{ccc}
\frac{8}{12} & -\frac{1}{12} & 0 \\
\frac{4}{3} & \frac{1}{3} & 0 \\
2 & \frac{5}{4} & 0
\end{array}\right]\left[\begin{array}{l}
f_{n+1} \\
f_{n+2} \\
f_{n+3}
\end{array}\right] } \\
& +\left[\begin{array}{ccc}
0 & 0 & \frac{5}{12} \\
0 & 0 & \frac{1}{3} \\
0 & 0 & -\frac{1}{4}
\end{array}\right]\left[\begin{array}{c}
f_{n-2} \\
f_{n-1} \\
f_{n}
\end{array}\right]
\end{aligned}
$$

where,

$$
A^{(0)}=\left[\begin{array}{lll}
1 & 0 & 0 \\
0 & 1 & 0 \\
0 & 0 & 1
\end{array}\right], A^{(1)}=\left[\begin{array}{lll}
0 & 0 & 1 \\
0 & 0 & 1 \\
0 & 0 & 1
\end{array}\right], B^{(0)}=\left[\begin{array}{ccc}
\frac{8}{12} & -\frac{1}{12} & 0 \\
\frac{4}{3} & \frac{1}{3} & 0 \\
2 & \frac{5}{4} & 0
\end{array}\right]
$$

and

$$
B^{(1)}=\left[\begin{array}{ccc}
0 & 0 & \frac{5}{12} \\
0 & 0 & \frac{1}{3} \\
0 & 0 & -\frac{1}{4}
\end{array}\right]
$$

The first characteristics polynomial of the scheme is

$$
\rho(\lambda)=\operatorname{det}\left[\lambda A^{0}-A^{1}\right]
$$




$$
\begin{gathered}
\rho(\lambda)=\operatorname{det}\left[\left(\begin{array}{lll}
\lambda & 0 & 0 \\
0 & \lambda & 0 \\
0 & 0 & \lambda
\end{array}\right)-\left(\begin{array}{lll}
0 & 0 & 1 \\
0 & 0 & 1 \\
0 & 0 & 1
\end{array}\right)\right] \\
=\operatorname{det}\left[\begin{array}{ccc}
\lambda & 0 & -1 \\
0 & \lambda & -1 \\
0 & 0 & \lambda-1
\end{array}\right] \\
\left|\begin{array}{lll}
\lambda & 0 & -1 \\
0 & \lambda & -1 \\
0 & 0 & \lambda-1
\end{array}\right|=0 \\
\lambda^{2}(\lambda-1)=0 \\
\lambda_{1}=\lambda_{2} \text { or } \lambda_{3}=1
\end{gathered}
$$

\subsection{Zero-Stability for $k=3$}

A block method is said to be stable as $h \rightarrow 0$ if the roots of the first characteristics polynomial defined by

$$
\rho \lambda=\operatorname{det}\left[\lambda A^{0}-A^{1}\right]
$$

satisfies $\left|r_{s}\right|=1$

The scheme can be expressed as

$$
\begin{aligned}
& {\left[\begin{array}{llll}
1 & 0 & 0 & 0 \\
0 & 1 & 0 & 0 \\
0 & 0 & 1 & 0 \\
0 & 0 & 0 & 1
\end{array}\right]\left[\begin{array}{l}
y_{n+1} \\
y_{n+2} \\
y_{n+3} \\
y_{n+4}
\end{array}\right]=\left[\begin{array}{llll}
0 & 0 & 0 & 1 \\
0 & 0 & 0 & 1 \\
0 & 0 & 0 & 1 \\
0 & 0 & 0 & 1
\end{array}\right]\left[\begin{array}{c}
y_{n-3} \\
y_{n-2} \\
y_{n-1} \\
y_{n}
\end{array}\right]} \\
& +h\left[\begin{array}{cccc}
\frac{89}{120} & -\frac{19}{120} & \frac{1}{40} & 0 \\
\frac{124}{90} & \frac{4}{15} & \frac{2}{45} & -\frac{1}{90} \\
\frac{77}{60} & \frac{23}{60} & \frac{-1}{60} & 0 \\
\frac{9}{8} & \frac{9}{8} & \frac{3}{8} & 0 \\
-\frac{7}{30} & \frac{47}{30} & \frac{17}{10} & 0
\end{array}\right]\left[\begin{array}{l}
f_{n+1} \\
f_{n+2} \\
f_{n+3} \\
f_{n+4}
\end{array}\right]+\left[\begin{array}{cccc}
0 & 0 & 0 & \frac{47}{120} \\
0 & 0 & 0 & \frac{7}{20} \\
0 & 0 & 0 & \frac{3}{8} \\
0 & 0 & 0 & \frac{29}{30}
\end{array}\right]\left[\begin{array}{l}
f_{n-3} \\
f_{n-2} \\
f_{n-1} \\
f_{n}
\end{array}\right]
\end{aligned}
$$

where,

$$
A^{(0)}=\left[\begin{array}{llll}
1 & 0 & 0 & 0 \\
0 & 1 & 0 & 0 \\
0 & 0 & 1 & 0 \\
0 & 0 & 0 & 1
\end{array}\right], A^{(1)}=\left[\begin{array}{llll}
0 & 0 & 0 & 1 \\
0 & 0 & 0 & 1 \\
0 & 0 & 0 & 1 \\
0 & 0 & 0 & 1
\end{array}\right], B^{(0)}=\left[\begin{array}{cccc}
\frac{89}{120} & -\frac{19}{120} & \frac{1}{40} & 0 \\
\frac{77}{60} & \frac{23}{60} & \frac{-1}{60} & 0 \\
\frac{9}{8} & \frac{9}{8} & \frac{3}{8} & 0 \\
-\frac{7}{30} & \frac{47}{30} & \frac{17}{10} & 0
\end{array}\right]
$$

and 


$$
B^{(1)}=\left[\begin{array}{cccc}
0 & 0 & 0 & \frac{47}{120} \\
0 & 0 & 0 & \frac{7}{20} \\
0 & 0 & 0 & \frac{3}{8} \\
0 & 0 & 0 & \frac{29}{30}
\end{array}\right]
$$

The first characteristics polynomial of the scheme is

$$
\begin{gathered}
\left.\rho(\lambda)=\operatorname{det}\left[\begin{array}{cccc}
\lambda & 0 & 0 & 0 \\
0 & \lambda & 0 & 0 \\
0 & 0 & \lambda & 0 \\
0 & 0 & 0 & \lambda
\end{array}\right)-\left(\begin{array}{llll}
0 & 0 & 0 & 1 \\
0 & 0 & 0 & 1 \\
0 & 0 & 0 & 1 \\
0 & 0 & 0 & 1
\end{array}\right)\right]=\operatorname{det}\left[\begin{array}{cccc}
\lambda & 0 & 0 & -1 \\
0 & \lambda & 0 & -1 \\
0 & 0 & \lambda-1 & 0 \\
0 & 0 & 0 & \lambda-1
\end{array}\right] \\
\qquad \begin{array}{llll}
\lambda & 0 & 0 & -1 \\
0 & \lambda & 0 & -1 \\
0 & 0 & \lambda & -1 \\
0 & 0 & 0 & \lambda-1
\end{array} \mid=0 \\
\lambda^{3}(\lambda-1)=0 \\
\lambda_{1}=\lambda_{2}=\lambda_{3}=0 \text { or } \lambda_{4}=1
\end{gathered}
$$

\subsection{Zero-Stability for $k=4$}

A block method is said to be stable as $h \rightarrow 0$ if the roots of the first characteristics polynomial defined by

$$
\rho \lambda=\operatorname{det}\left[\lambda A^{0}-A^{1}\right]
$$

satisfies $\left|r_{s}\right|=1$

The scheme can be expressed as

$$
\begin{aligned}
& {\left[\begin{array}{lllll}
1 & 0 & 0 & 0 & 0 \\
0 & 1 & 0 & 0 & 0 \\
0 & 0 & 1 & 0 & 0 \\
0 & 0 & 0 & 1 & 0 \\
0 & 0 & 0 & 0 & 1
\end{array}\right]\left[\begin{array}{l}
y_{n+1} \\
y_{n+2} \\
y_{n+3} \\
y_{n+4} \\
y_{n+5}
\end{array}\right]=\left[\begin{array}{lllll}
0 & 0 & 0 & 0 & 1 \\
0 & 0 & 0 & 0 & 1 \\
0 & 0 & 0 & 0 & 1 \\
0 & 0 & 0 & 0 & 1 \\
0 & 0 & 0 & 0 & 1
\end{array}\right]\left[\begin{array}{c}
y_{n-4} \\
y_{n-3} \\
y_{n-2} \\
y_{n-1} \\
y_{n}
\end{array}\right]} \\
& +h\left[\begin{array}{ccccc}
\frac{56150}{144} & -\frac{84084}{144} & \frac{56042}{144} & -\frac{14009}{144} & 0 \\
\frac{124}{90} & \frac{4}{15} & \frac{2}{45} & -\frac{1}{90} & 0 \\
\frac{754}{560} & \frac{444}{560} & \frac{334}{560} & -\frac{31}{560} & 0 \\
\frac{64}{45} & \frac{8}{9} & \frac{64}{45} & \frac{14}{45} & 0 \\
\frac{3322}{1008} & -\frac{1308}{1008} & \frac{1222}{1008} & \frac{2057}{1008} & 0
\end{array}\right]\left[\begin{array}{l}
f_{n+1} \\
f_{n+2} \\
f_{n+3} \\
f_{n+4} \\
f_{n+5}
\end{array}\right]
\end{aligned}
$$




$$
+\left[\begin{array}{ccccc}
0 & 0 & 0 & 0 & -\frac{13955}{144} \\
0 & 0 & 0 & 0 & \frac{29}{90} \\
0 & 0 & 0 & 0 & \frac{179}{560} \\
0 & 0 & 0 & 0 & \frac{14}{45} \\
0 & 0 & 0 & 0 & -\frac{253}{1008}
\end{array}\right]\left[\begin{array}{c}
f_{n-4} \\
f_{n-3} \\
f_{n-2} \\
f_{n-1} \\
f_{n}
\end{array}\right]
$$

where,

$$
\begin{aligned}
& A^{(0)}=\left[\begin{array}{lllll}
1 & 0 & 0 & 0 & 0 \\
0 & 1 & 0 & 0 & 0 \\
0 & 0 & 1 & 0 & 0 \\
0 & 0 & 0 & 1 & 0 \\
0 & 0 & 0 & 0 & 1
\end{array}\right], A^{(1)}=\left[\begin{array}{ccccc}
0 & 0 & 0 & 0 & 1 \\
0 & 0 & 0 & 0 & 1 \\
0 & 0 & 0 & 0 & 1 \\
0 & 0 & 0 & 0 & 1 \\
0 & 0 & 0 & 0 & 1
\end{array}\right], \\
& B^{(0)}=\left[\begin{array}{ccccc}
\frac{56150}{144} & -\frac{84084}{144} & \frac{56042}{144} & -\frac{14009}{144} & 0 \\
\frac{124}{90} & \frac{4}{15} & \frac{2}{45} & -\frac{1}{90} & 0 \\
\frac{754}{560} & \frac{444}{560} & \frac{334}{560} & -\frac{31}{560} & 0 \\
\frac{64}{45} & \frac{8}{9} & \frac{64}{45} & \frac{14}{45} & 0 \\
\frac{3322}{1008} & -\frac{1308}{1008} & \frac{1222}{1008} & \frac{2057}{1008} & 0
\end{array}\right]
\end{aligned}
$$

and

$$
B^{(1)}=\left[\begin{array}{ccccc}
0 & 0 & 0 & 0 & -\frac{13955}{144} \\
0 & 0 & 0 & 0 & \frac{29}{90} \\
0 & 0 & 0 & 0 & \frac{179}{560} \\
0 & 0 & 0 & 0 & \frac{14}{45} \\
0 & 0 & 0 & 0 & -\frac{253}{1008}
\end{array}\right]
$$

The first characteristics polynomial of the scheme is

$$
\begin{gathered}
\rho(\lambda)=\operatorname{det}\left[\lambda A^{0}-A^{1}\right] \\
\rho(\lambda)=\operatorname{det}\left[\left(\begin{array}{cccc}
\lambda & 0 & 0 & 0 \\
0 & \lambda & 0 & 0 \\
0 & 0 & \lambda & 0 \\
0 & 0 & 0 & \lambda
\end{array}\right)-\left(\begin{array}{llll}
0 & 0 & 0 & 1 \\
0 & 0 & 0 & 1 \\
0 & 0 & 0 & 1 \\
0 & 0 & 0 & 1
\end{array}\right)\right]=\operatorname{det}\left[\begin{array}{cccc}
\lambda & 0 & 0 & -1 \\
0 & \lambda & 0 & -1 \\
0 & 0 & \lambda-1 & 0 \\
0 & 0 & 0 & \lambda-1
\end{array}\right]
\end{gathered}
$$




$$
\begin{gathered}
\left|\begin{array}{ccccc}
\lambda & 0 & 0 & 0 & -1 \\
0 & \lambda & 0 & 0 & -1 \\
0 & 0 & \lambda & 0 & -1 \\
0 & 0 & 0 & \lambda & -1 \\
0 & 0 & 0 & 0 & \lambda-1
\end{array}\right|=0 \\
\lambda^{4}(\lambda-1)=0 \\
\lambda_{1}=\lambda_{2}=\lambda_{3}=\lambda_{4}=0 \text { or } \lambda_{5}=1
\end{gathered}
$$

\section{Numerical Experiments}

In order to confirm the accuracy and efficiency of the scheme, we consider the following initial value problems: Tables 1-4.

Table 1. Results and errors of problem 1 for $K=3$.

\begin{tabular}{ccccc}
\hline$N$ & $X$ & YC & YEX & $\mid$ YEX - YC $\mid$ \\
\hline 0 & 0.000000 & 1.000000 & 1.000000 & 0.000000 \\
1 & 0.100000 & 0.904874 & 0.904837 & $3.7 \times 10^{-5}$ \\
2 & 0.200000 & 0.818800 & 0.818730 & $7.0 \times 10^{-5}$ \\
3 & 0.300000 & 0.740889 & 0.740818 & $7.1 \times 10^{-5}$ \\
4 & 0.400000 & 0.670418 & 0.670320 & $9.8 \times 10^{-5}$ \\
5 & 0.500000 & 0.606653 & 0.606531 & $1.22 \times 10^{-4}$ \\
6 & 0.600000 & 0.548956 & 0.548811 & $1.45 \times 10^{-4}$ \\
7 & 0.700000 & 0.496749 & 0.496585 & $1.64 \times 10^{-4}$ \\
8 & 0.800000 & 0.449511 & 0.449328 & $1.83 \times 10^{-4}$ \\
9 & 0.900000 & 0.406768 & 0.406569 & $1.99 \times 10^{-4}$ \\
10 & 1.000000 & 0.368093 & 0.367879 & $2.14 \times 10^{-4}$ \\
\hline
\end{tabular}

Table 2. Results and errors of problem 1 for $K=4$.

\begin{tabular}{ccccc}
\hline$N$ & $X$ & YC & YEX & $\mid$ YEX - YC $\mid$ \\
\hline 0 & 0.000000 & 1.000000 & 1.000000 & 0.000000 \\
1 & 0.100000 & 0.905695 & 0.904837 & $8.58 \times 10^{-4}$ \\
2 & 0.200000 & 0.820365 & 0.818730 & $1.635 \times 10^{-3}$ \\
3 & 0.300000 & 0.743155 & 0.740818 & $2.337 \times 10^{-3}$ \\
4 & 0.400000 & 0.673292 & 0.670320 & $2.972 \times 10^{-3}$ \\
5 & 0.500000 & 0.610078 & 0.606531 & $3.547 \times 10^{-3}$ \\
6 & 0.600000 & 0.552879 & 0.548811 & $4.068 \times 10^{-3}$ \\
7 & 0.700000 & 0.501124 & 0.496585 & $4.539 \times 10^{-3}$ \\
8 & 0.800000 & 0.463649 & 0.449328 & $1.4321 \times 10^{-2}$ \\
9 & 0.900000 & 0.429739 & 0.406569 & $2.3170 \times 10^{-2}$ \\
10 & 1.000000 & 0.399057 & 0.367879 & $3.1178 \times 10^{-2}$ \\
\hline
\end{tabular}


Table 3. Results and errors of problem 2 for $K=3$.

\begin{tabular}{ccccc}
\hline$N$ & $X$ & YC & YEX & $\mid$ YEX $-\mathrm{YC} \mid$ \\
\hline 0 & 0.000000 & 0.000000 & 0.000000 & 0.000000 \\
1 & 0.100000 & 0.004993 & 0.004988 & $5.0 \times 10^{-6}$ \\
2 & 0.200000 & 0.019806 & 0.019801 & $5.0 \times 10^{-6}$ \\
3 & 0.300000 & 0.044003 & 0.044003 & 0.00000 \\
4 & 0.400000 & 0.076890 & 0.076884 & $6.0 \times 10^{-6}$ \\
5 & 0.500000 & 0.117509 & 0.117503 & $6.0 \times 10^{-6}$ \\
6 & 0.600000 & 0.164732 & 0.164729 & $3.0 \times 10^{-6}$ \\
7 & 0.700000 & 0.217303 & 0.217295 & $8.0 \times 10^{-6}$ \\
8 & 0.800000 & 0.273858 & 0.273851 & $7.0 \times 10^{-6}$ \\
9 & 0.900000 & 0.333026 & 0.333023 & $3.0 \times 10^{-6}$ \\
10 & 1.000000 & 0.393477 & 0.393469 & $8.0 \times 10^{-6}$ \\
\hline
\end{tabular}

Table 4. Results and errors of problem 2 for $K=4$.

\begin{tabular}{ccccc}
\hline$N$ & $X$ & YC & YEX & $\mid$ YEX - YC $\mid$ \\
\hline 0 & 0.000000 & 0.000000 & 0.000000 & 0.000000 \\
1 & 0.100000 & 0.004434 & 0.004988 & $5.5 \times 10^{-4}$ \\
3 & 0.200000 & 0.019809 & 0.019801 & $8.0 \times 10^{-6}$ \\
4 & 0.300000 & 0.044009 & 0.044003 & $6.0 \times 10^{-6}$ \\
5 & 0.400000 & 0.076891 & 0.076884 & $7.0 \times 10^{-6}$ \\
6 & 0.500000 & 0.129887 & 0.117503 & $1.2 \times 10^{-2}$ \\
7 & 0.600000 & 0.168705 & 0.164729 & $3.9 \times 10^{-3}$ \\
8 & 0.700000 & 0.216646 & 0.217295 & $6.5 \times 10^{-4}$ \\
9 & 0.800000 & 0.272938 & 0.273851 & $9.1 \times 10^{-4}$ \\
10 & 0.900000 & 0.344677 & 0.333023 & $1.1 \times 10^{-2}$ \\
\hline
\end{tabular}

Problem 1:

$$
y^{\prime}=-y(x), h=0.1, y(0)=1
$$

Exact solution: $y(x)=\mathrm{e}^{-x} \quad$ (see K.M. Abualnaja, 2015).

YC: approximate solution

YEX: exact solution

\section{Problem 2:}

$$
y^{\prime}(x)=-x(1-y), h=0.1, y(0)=0
$$

Exact solution: $y(x)=1-\mathrm{e}^{-\frac{x^{2}}{2}} \quad$ (see K.M. Abualnaja, 2015).

YC: approximate solution

YEX: exact solution 


\section{Conclusion}

In this research work, a class of implicit block collocation methods for the direct solution of initial value problems of general first order ordinary differential equations was developed using Legendre collocation approach. The collocation technique yielded a consistent and zero stable implicit block multi-step method with continuous coefficients. The method is implemented without the need for the development of correctors.

\section{References}

[1] Lambert, J.D. (1973) Computational Methods in Ordinary Differential Equations. John Wiley and Sons, New York, 278.

[2] Fatunla, S.O. (1988) Numerical Methods for Initial Value Problems in Ordinary Differential Equations. Academic Press, New York, 398 p.

[3] Sararfyan, D. (1990) New Algorithm for the Continuous Approximate Solutions of ODEs. Journal of Computer and Mathematics, 20, 77-100.

[4] Awoyemi, D.O. (2001) A New Sixth-Order Algorithm for General Second Order ODE. International Journal of Computational Mathematics, 77, 117-124.

[5] Kayode, S.J. (2008) An Efficient Zero-Stable Numerical Method for Fourth-Order Differential Equations. International Journal of Mathematics and Mathematical Sciences, 1-10.

[6] Hairer, E. and Wanner, G. (1975) A Theory for Nystrom Methods. Numerical Mathematics, 25, 383-400. https://doi.org/10.1007/BF01396335

[7] Henrici, P. (1962) Discrete Variable Methods in ODE. John Wiley \& Sons, New York, 729p.

[8] Awoyemi, D.O. (2005) Algorithm Collocation Approach for Direct Solution of Fourth Order Initial Value Problems of ODEs. International Journal of Computational Mathematics, 82, 271-284.

[9] Adee, S.O., Onumanyi, P., Sirisena, U.W. and Yahaya, Y.A. (2005) Note on Starting the Numerov Method More Accurately by a Hybrid Formula of Order Four for Initial Value Problems. Journal of Computational and Applied Mathematics, 175, 369-373. https://doi.org/10.1016/j.cam.2004.06.016

[10] Yahaya, A.M. and Badmus, Y.A. (2009) An Accurate Uniform Order 6, Block Method for Direct Solution of General Second Order Ordinary Differential Equation. Pacific Journal of Science and Technology, 10, 248-254.

[11] Milne, W.E. (1953) Numerical Solution of Differential Equations. Wiley, New York, $456 \mathrm{p}$.

[12] Chu, M.T. and Hamilton, H. (1987) Parallel Solution of ODEs by Multiblock Methods. SIAM Journal of Scientific and Statistical Computation, 8, 342-553. https://doi.org/10.1137/0908039

[13] Omar, Z.B. and Suleiman, M.B. (1999) Solving Second Order ODEs Directly Using Parallel 2-point Explicit Block Method. Mathematical, 21, 15-23.

[14] Omar, Z. and Suleiman, M. (2003) Parallel R-Point Implicit Block Method for Solving Higher Order Ordinary Differential Equation Directly. Journal of Information Communication Technology, 3, 53-66.

[15] Omar, Z.B. and Suleiman, M.B. (2005) Solving Higher Order ODEs Directly Using Parallel 2-Point Explicit Block Method. Mathematical, 21, 51-72. 
[16] Areo, E.A. and Adeniyi, R.B. (2013) Sixth Order Hybrid Block for the Numerical Solution of First Order Initial Value Problems. Mathematical Theory and Modelling, 82, 345-364. 